\title{
Erratum to: SUSY Background to Neutral MSSM Higgs Boson Searches
}

\author{
B. Feigl ${ }^{1, \mathrm{a}}$, H. Rzehak ${ }^{2, \mathrm{~b}}$, D. Zeppenfeld ${ }^{1, \mathrm{c}}$ \\ ${ }^{1}$ Institut für Theoretische Physik, Karlsruher Institut für Technologie, 76128 Karlsruhe, Germany \\ ${ }^{2} \mathrm{PH}-\mathrm{TH}, \mathrm{CERN}, 1211$ Geneva 23, Switzerland
}

Received: 25 July 2013 / Revised: 26 August 2013 / Published online: 13 September 2013

(C) Springer-Verlag Berlin Heidelberg and Società Italiana di Fisica 2013

\section{Erratum to: Eur. Phys. J. C (2012) 72:1903 DOI 10.1140/epjc/s10052-012-1903-7}

We correct an error in the calculation of the signal process $p p \rightarrow h j j \rightarrow W^{+} W^{-} j j \rightarrow \ell^{+} \ell^{-} \nu_{\ell} \bar{\nu}_{\ell} j j$ in vector boson fusion. Some lepton distributions were wrong, which affects the cross section after cuts. However, the conclusions remain unchanged.

In this erratum we correct the signal process estimates from Ref. [1] for the process

$$
p p \rightarrow h j j \rightarrow W^{+} W^{-} j j \rightarrow \ell^{+} \ell^{-} \nu_{\ell} \bar{\nu}_{\ell} j j
$$

in vector boson fusion. For simplicity we use the same notation as in Ref. [1].

Version 2.5 of VBFNLO [2, 3], which was used to generate the signal process, interchanged the momenta of $\ell^{-}$ and $\bar{v}_{\ell}$ for process ID 105 and thus produced wrong $m_{\ell \ell}$, $\phi_{\ell \ell}, p_{T, \ell \ell}, m_{T}(W W)$ and $m_{\tau \tau, r e c}$ distributions, which led to an underestimation of the signal process cross section by roughly $40 \%$ (for $\sqrt{s}=14 \mathrm{TeV}$ ), once the cuts of Eq. (37) were applied. For this erratum we recalculated the signal cross section with VBFNLO 2.6.2, where this error has been fixed.

Signal cross sections affected by this error were included in Tables 5-10,12, 15 and 17-21 of the original paper. Those numbers also get quoted in the text of Sects. 4.3 and 6.1. Table 1 lists the corrected values in all discussed scenarios for the rows and columns labeled "VBF

The online version of the original article can be found under doi:10.1140/epjc/s10052-012-1903-7.

\footnotetext{
a e-mail: bastian.feigl@kit.edu

b e-mail: heidi.rzehak@cern.ch

c e-mail: dieter.zeppenfeld@kit.edu
}

$h \rightarrow W W$ " of these tables. The relative size of the background quoted at the end of Sect. 4.2.1 decreases to $12 \%$ of the $h \rightarrow W W$ cross section, for the scenario in Sect. 4.2.2 it drops to $6.4 \%$ and in the beginning of Sect. 8.2 the correct number is $12 \%$.

Tables 12, 15 and 17-21 of Ref. [1] furthermore list the ratio of signal to SUSY background $S / B^{\mathrm{SUSY}}$, which is affected by the wrong signal cross sections. The wrong results of $S / B^{\text {SUSY }}$ also get referenced in Eqs. (46), (47), (50)-(52), (54), in the text right after Eq. (51) and in the third-last paragraph of the conclusion. In Table 2 we list the corrected values for $S / B^{\text {SUSY }}$ in all discussed scenarios.

The signal cross sections given in the text of Sect. 6.3 change from $0.76 \mathrm{fb}$ to $1.33 \mathrm{fb}(1.08 \mathrm{fb}$ to $1.98 \mathrm{fb})$ for small (large) stop/sbottom masses. Furthermore, for the scenario with a Higgs boson mass of $124.3 \mathrm{GeV}$ the corrected signal cross section is 527 ab, giving $S / B^{\mathrm{SUSY}}=8.4$ (Eq. (55)).

The cross sections entering in the calculation of the Central Jet Veto probability for the $h \rightarrow W W$ signal process change, but the veto probability increases only slightly. Table 3 contains the corrected values for Table 7 of Ref. [1].

Several figures in the original analysis showing the $h \rightarrow W W$ distributions are affected by the error. However, changes for most distributions can be well described by rescaling the VBF $h \rightarrow W W$ curve with the correction factor of the $h \rightarrow W W$ cross section. The sole distribution where not only the signal normalization but also the signal shape changes substantially is the $m_{\ell \ell}$ distribution in Fig. 16. The corrected distribution is given in Fig. 1.

As the signal cross section increased with respect to the originally stated numbers, the conclusion of the original paper remains unchanged: The SUSY background processes to VBF Higgs boson production with a subsequent decay into 
Table 1 Total cross sections for VBF $h \rightarrow W W$ at different cut levels for all discussed scenarios

\begin{tabular}{|c|c|c|c|c|c|c|}
\hline \multirow[t]{2}{*}{ Cuts } & \multicolumn{4}{|c|}{ LHC energy $\sqrt{s}=14 \mathrm{TeV}$} & \multicolumn{2}{|c|}{ LHC energy $\sqrt{s}=7 \mathrm{TeV}$} \\
\hline & $\begin{array}{l}\text { SPS1amod/ } \\
m_{\tilde{q}} \approx 1.1 \mathrm{TeV} / \\
\text { light sleptons }\end{array}$ & SPS1amod2 & SPS1a-slope & $\begin{array}{l}\text { Light sleptons } \\
+ \text { higher } m_{\widetilde{q}}, m_{\widetilde{g}}\end{array}$ & SPS1amod & $\begin{array}{l}\text { Light sleptons } \\
+ \text { higher } m_{\widetilde{q}}, m_{\widetilde{g}}\end{array}$ \\
\hline Eq. (35) & $5.09 \mathrm{fb}$ & - & - & - & - & - \\
\hline + Eq. (36) & $2.91 \mathrm{fb}$ & $4.96 \mathrm{fb}$ & $5.32 \mathrm{fb}$ & $4.50 \mathrm{fb}$ & $777 \mathrm{ab}$ & $1193 \mathrm{ab}$ \\
\hline + Eq. (37) & $1.46 \mathrm{fb}$ & $2.48 \mathrm{fb}$ & - & - & - & - \\
\hline + Eq. (39) & $1.37 \mathrm{fb}$ & $2.28 \mathrm{fb}$ & - & - & - & - \\
\hline + Eq. (40) & $1.33 \mathrm{fb}$ & $2.21 \mathrm{fb}$ & - & - & - & - \\
\hline+ Eq. (42) & $1.32 \mathrm{fb}$ & - & $2.37 \mathrm{fb}$ & $2.00 \mathrm{fb}$ & $316 \mathrm{ab}$ & $476 \mathrm{ab}$ \\
\hline+ Eq. (45) & $1.32 \mathrm{fb}$ & - & $2.37 \mathrm{fb}$ & $2.00 \mathrm{fb}$ & $316 a b$ & $476 \mathrm{ab}$ \\
\hline $\begin{array}{l}\text { Referenced in } \\
\text { original Table }\end{array}$ & $\begin{array}{l}5,8,9,10 \\
12,15,18\end{array}$ & 6 & 17 & 19 & 20 & 21 \\
\hline
\end{tabular}

Table 2 Signal over SUSY background ratio $S / B^{\text {SUSY }}$, with VBF $h \rightarrow W W$ as signal. SUSY background contributions considered include the production of $\chi_{1}^{+} \chi_{1}^{-} j j, \chi_{2}^{0} \chi_{1}^{0} j j, \widetilde{\ell^{+}} \widetilde{\ell}^{-} j j, \chi_{1}^{ \pm} \chi_{2}^{0} j j$ and $\chi_{2}^{0} \chi_{2}^{0} j j$. Values for all discussed scenarios of [1] are listed

\begin{tabular}{|c|c|c|c|c|c|c|}
\hline Processes & $\sqrt{s}$ & $\begin{array}{l}\text { Original } \\
\text { Table }\end{array}$ & $\begin{array}{l}\text { Basic cuts } \\
\text { Eq. }(35)\end{array}$ & $\begin{array}{l}\text { + rap. gap } \\
+(36)\end{array}$ & $\begin{array}{l}+m_{i n v}, \not p_{T}, \phi_{\ell \ell}, \mathrm{b}-\mathrm{tag} \\
+(37),(39),(40),(42)\end{array}$ & $\begin{array}{l}+ \text { CJV on } j_{\text {decay }} \\
+(45)\end{array}$ \\
\hline SPS1amod & $14 \mathrm{TeV}$ & 12 & 0.015 & 0.25 & 1.3 & 1.9 \\
\hline$m_{\tilde{q}} \approx 1.1 \mathrm{TeV}$ & $14 \mathrm{TeV}$ & 15 & - & 0.60 & 4.9 & 6.2 \\
\hline SPS1a-slope & $14 \mathrm{TeV}$ & 17 & - & 1.4 & 12 & 18 \\
\hline Light sleptons & $14 \mathrm{TeV}$ & 18 & - & 0.046 & 0.69 & 0.69 \\
\hline Light sleptons + higher $m_{\widetilde{q}}, m_{\widetilde{g}}$ & $14 \mathrm{TeV}$ & 19 & - & 0.23 & 3.9 & 3.9 \\
\hline SPS1amod & $7 \mathrm{TeV}$ & 20 & - & 0.52 & 2.5 & 3.6 \\
\hline Light sleptons + higher $m_{\tilde{q}}, m_{\tilde{g}}$ & $7 \mathrm{TeV}$ & 21 & - & 0.44 & 8.1 & 8.1 \\
\hline
\end{tabular}

Table 3 Total cross sections and central jet veto probabilities for $\chi_{1}^{+} \chi_{1}^{-} j j(j), \chi_{2}^{0} \chi_{1}^{0} j j(j)$ and $h \rightarrow W W$ with final cuts. For the $j j j$ case, the cross sections are within the veto region from Eq. (41)

\begin{tabular}{llll}
\hline$\sigma$ & $j j$ & $j j j$ & $P_{\text {veto }}$ \\
\hline$\chi_{1}^{+} \chi_{1}^{-}$ & $0.073 \mathrm{fb}$ & $0.044 \mathrm{fb}$ & 0.45 \\
$\chi_{2}^{0} \chi_{1}^{0}$ & $0.081 \mathrm{fb}$ & $0.109 \mathrm{fb}$ & 0.74 \\
$h \rightarrow W W$ & $1.38 \mathrm{fb}$ & $0.139 \mathrm{fb}$ & 0.10 \\
\hline
\end{tabular}

W-bosons or tau-leptons are under control for squark and gluino masses at or above $1.1 \mathrm{TeV}$.

\section{References}

1. B. Feigl, H. Rzehak, D. Zeppenfeld, Eur. Phys. J. C 72, 1903 (2012). arXiv:1108.1110 [hep-ph]

2. K. Arnold et al., Comput. Phys. Commun. 180, 1661-1670 (2009). arXiv:0811.4559 [hep-ph]

3. K. Arnold et al., arXiv:1107.4038 [hep-ph]

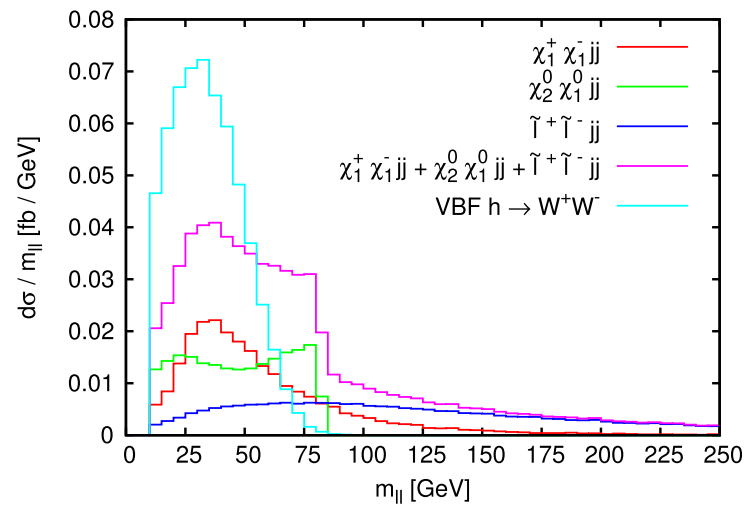

Fig. 1 Invariant lepton pair mass distribution of the $\tilde{\ell}^{+} \tilde{\ell}^{-} j j$, $\chi_{1}^{+} \chi_{1}^{-} j j$ and $\chi_{2}^{0} \chi_{1}^{0} j j$ background channel, of the sum of the considered background channels and the signal process. Cuts of Eqs. (35) and (36) are applied, b-quark contributions are not included 